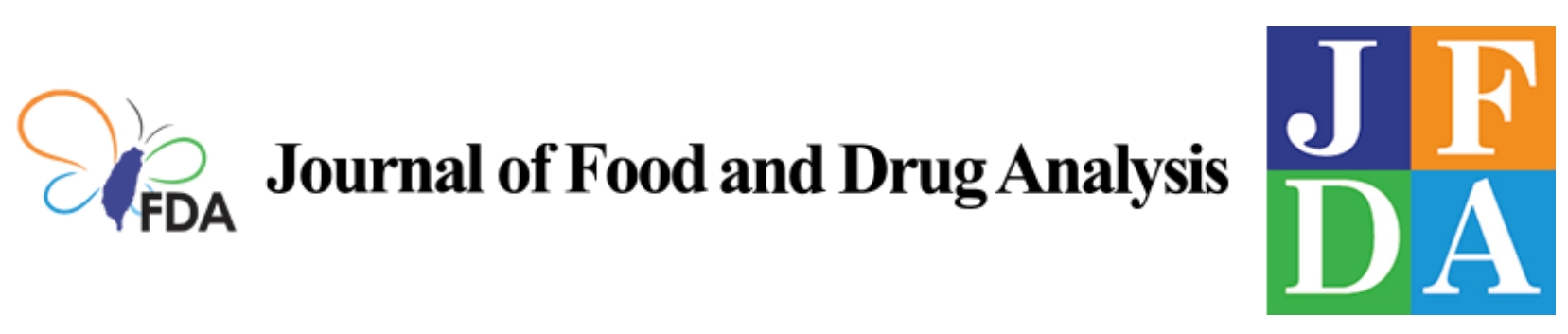

Volume 28 | Issue 2

Article 8

2020

\title{
A rapid strategy for screening high-efficiency PCSK9 inhibitors from Ginkgo biloba leaves by ligand fishing, HPLC-Q-TOF-MS and interdisciplinary assay
}

Follow this and additional works at: https://www.jfda-online.com/journal

Part of the Food Science Commons, Medicinal Chemistry and Pharmaceutics Commons, Pharmacology Commons, and the Toxicology Commons

(c) (i) (9)

This work is licensed under a Creative Commons Attribution-Noncommercial-No Derivative Works 4.0 License.

\section{Recommended Citation}

Li, Li; Fan, Meng-Lin; Li, Ya-Nan; Huang, Ya-Xuan; Liu, Xiu-Feng; and Liu, Ji-Hua (2020) "A rapid strategy for screening high-efficiency PCSK9 inhibitors from Ginkgo biloba leaves by ligand fishing, HPLC-Q-TOF-MS and interdisciplinary assay," Journal of Food and Drug Analysis: Vol. 28 : Iss. 2 , Article 8.

Available at: https://doi.org/10.38212/2224-6614.1061

This Original Article is brought to you for free and open access by Journal of Food and Drug Analysis. It has been accepted for inclusion in Journal of Food and Drug Analysis by an authorized editor of Journal of Food and Drug Analysis. 


\title{
A rapid strategy for screening high-efficiency PCSK9 inhibitors from Ginkgo biloba leaves by ligand fishing, HPLC-Q-TOF-MS and interdisciplinary assay
}

\author{
Li Li $^{\text {a,c }}$, Meng-Lin Fan ${ }^{a}$, Ya-Nan Li ${ }^{a}$, Ya-Xuan Huang ${ }^{a}$, Xiu-Feng Liu ${ }^{\text {a,**, }}$ Ji-Hua Liu ${ }^{\text {a,b, } *}$
}

a Jiangsu Key Laboratory of TCM Evaluation and Translational Research, School of Traditional Chinese Pharmacy, China Pharmaceutical University, Nanjing, Jiangsu 211198, PR China

b State Key Laboratory of Natural Medicines, China Pharmaceutical University, Nanjing, Jiangsu 211198, PR China

c School of Pharmacy, Hebei Medical University, Shijiazhuang 050017, PR China

\begin{abstract}
Proprotein convertase subtilisin/kexin type 9 (PCSK9) is an attractive target for new cholesterol-lowering drug development. Here, we developed a method integrating ligand fishing, HPLC-Q-TOF-MS and interdisciplinary assay, aiming to explore potential PCSK9 inhibitors from mixtures rapidly and accurately. PCSK9 was expressed and purified firstly, and then the recombined PCSK9 was coated on the surface of magnetic beads (MBs). The PCSK9-immobilized MBs (PCSK9-MBs) were used for ligand fishing combined with HPLC and Q-TOF-MS/MS. Ginkgo biloba leaves (GBL), an herbal medicine widely used in Asia and Europe with good efficacy in treatment of hypercholesterolemia, were chosen as an illustration for ligand fishing. Two PCSK9 ligands were discovered from GBL and identified as kaempferol3-O-rutinoside (1) and kaempferol 3-O-2"'-(6"' -p-coumaroyl) glucosylrhamnoside (KCGR) (2). In order to verify fishing results and pick out more powerful PCSK9 inhibitors, molecular docking assay was further performed and KCGR was optimized to be an excellent PCSK9 inhibitor by the confirmation of affinity and activity bioassay. These results suggested that the developed approach could be applied to screen and analysis potential bioactive constituents from mixtures, which may improve the efficiency of drug discovery. Moreover, KCGR separated from GBLwas expected to be a potential candidate of PCSK9 inhibitors.
\end{abstract}

Keywords: Drug discovery, Ginkgo biloba leaves, Hypercholesterolemia, Ligand fishing, PCSK9 inhibitor

\section{Introduction}

$\mathrm{T}$ he hyperlipidemia is one of the risk factors for cardiovascular disease, coronary atherosclerosis and stroke [1], causing prominent global adult mortality. Currently, statin drugs were the preferred choices for treating hyperlipidemia [2]. However, some patients suffered from familial hypercholesterolemia (FH) did not achieve the desired therapeutic effect even at maximal statin dose $[3,4]$. The low-density lipoprotein receptor (LDLR), which is expressed on the membrane surface of hepatocytes, can bind to the low-density lipoprotein cholesterol (LDL-C) [5]. Then, the complex of the LDLR/LDL-C collectively internalizes, after which the LDL-C is released and degraded into lipids and amino acids. Whereafter, LDLR retures to the cell surface, which is known as the recycling of LDLR [6]. However, the proprotein convertase subtilisin/kexin type 9 (PCSK9) can couple with the cell-surface LDLR and prevents its recycling, which promotes the degradation of LDLR, and consequently causing raised levels of plasma LDL-C in the patients with

* Corresponding author at: Jiangsu Key Laboratory of TCM Evaluation and Translational Research, School of Traditional Chinese Pharmacy, China Pharmaceutical University, Nanjing, Jiangsu 211198, PR China. Fax: +0086 02586185158.

** Corresponding author at: Jiangsu Key Laboratory of TCM Evaluation and Translational Research, School of Traditional Chinese Pharmacy, China Pharmaceutical University, Nanjing, Jiangsu 211198, PR China. Fax: + 008602586185158.

E-mail addresses: xf.liu@cpu.edu.cn (X.-F. Liu), liujihua@cpu.edu.cn (J.-H. Liu). 
FH $[7,8]$. Many researches have indicated that PCSK9 inhibitors could efficiently decrease the plasma level of LDL-C by interrupting the protein-protein interaction of PCSK9/LDLR [5]. Therefore, searching for PCSK9 inhibitors would be a new and imperative therapy for the development of cholesterol-lowing drugs. Although three PCSK9 antibody-drugs have been approved $[9,10]$, the high cost and low compliance of antibody-drugs still caused the patients distressed. Considering lower cost and ease of administration, oral PCSK9 inhibitors have been an alternative therapy desirable for development.

Ginkgo biloba leaves (GBL) agents are the most widely used herbal supplements in recent years $[11,12]$. Previous studies have indicated that GBL had significantly lipid-regulating effects on hyperlipidemia [13-15]. However, the mechanism of the interaction between GBL and PCSK9, as well as the main active compounds of GBL contributing to the hypolipidemic effects remained unclear.

Taking advantages of good selectivity and high efficiency, several affinity-based strategies such as cell membrane chromatography [16], affinity ultrafiltration $[17,18]$ and molecular docking [19], have been frequently applied for screening bioactive compounds in herbal medicines. Ligand fishing is a welldeveloped affinity-based method in which ligands can be separated from unbound components in mixtures by selective binding to target enzymes or receptors. In consideration of preservation for protein activity and convenience in operating, magnetic beads (MBs) have been extensively served as solid supports for ligand fishing. Up to now, several researches have been reported to use enzyme/protein immobilized MBs to identify active compounds from the herbal medicines, such as inhibitors of ACE [20] and $\alpha$-glucosidase [21]. However, to the best of our knowledge, magnetic fishing-LC-MS (MF-LC-MS) method has not been reported for seeking PCSK9 ligands in herbal medicines or other complex mixtures.

In this study, a fishing strategy was established integrating MF-LC-MS and multidisciplinary activity detection to rapidly screen compounds for inhibiting PCSK9 from a complex natural extract (as shown in Fig. 1). PCSK9 was primarily immobilized onto the surface of MBs. MBs immobilized with PCSK9 (PCSK9-MBs) were then incubated with natural extracts to catch the potential PCSK9 ligands. Then, the unbound components were abandoned by magnetic separation and washing. The ligand-target complexes were obtained, and the bound components could be dissociated from the target by adding organic denature reagents. The dissociated components were analyzed by LC/MS. The interaction between PCSK9 and the potential ligands would be rapidly predicted by molecular docking to eliminate non-specific binding in the fishing period. Moreover, cell assays combined with bio-layer interferometry (BLI) binding assays were further conducted to find out potential inhibitors of the PCSK9 from the above ligands. The feasibility of this method was evaluated by a famous herbal medicine GBL for screening potential PCSK9 inhibitors.

\section{Methods}

\subsection{Apparatus, chemicals and reagents}

The Ginkgo biloba leaves (GBL) were purchased from Tong Ren Tang Industrial Corporation (Nanjing, China). The Ginkgo biloba leaves (GBL) were dried, powdered. Voucher specimens of GBL were identified and stored at $4{ }^{\circ} \mathrm{C}$ in Jiangsu Key Laboratory of TCM Evaluation and Translational Research, China Pharmaceutical University.

The reference standard of kaempferol-3-O-rutinoside was provided by Chengdu Herbpurify Co., Ltd (Chengdu, China). Formic acid of HPLC grade was purchased from Aladdin Industrial Corporation (Shanghai, China). Water was prepared by Milli-Q water purification system (Millipore, Bedford, United States). Methanol and acetonitrile (ACN) of HPLC grade were provided by Merck Company Inc. (Darmstadt, Germany). All other chemicals and solvents were of analytical grade.

Plasmid isolation and DNA purification kits were purchased from Transgene (Beijing, China). Isopropylthio- $\beta$-D-galactopyranoside (IPTG) was provided by Invitrogen (Beijing, China). Ni-NTA affinity resin was provided by Beyotime (Nanjing, China). Amicon Ultra Centrifugal Filter Units (30 kDa) were provided by Millipore (Bedford, United States).

\subsection{Preparation of PCSK9 coated magnetic beads}

The preparation of PCSK9-MBs was carried out according to our previous study with some alterations [22]. The human PCSK9 gene (152-692) was codon-optimized to permit bacterial expression, and the final PCSK9 sequence was inserted into a pET22b vector with the NdeI and XhoI sites, followed by transformation into Escherichia coliBL21 (DE3). Whereafter, the cells were incubated in TB medium supplemented with $50 \mu \mathrm{g} / \mathrm{mL}$ ampicillin at $37{ }^{\circ} \mathrm{C}$. The recombinant protein expression was induced by $0.1 \mathrm{mM}$ IPTG and incubated at $20{ }^{\circ} \mathrm{C}$ 


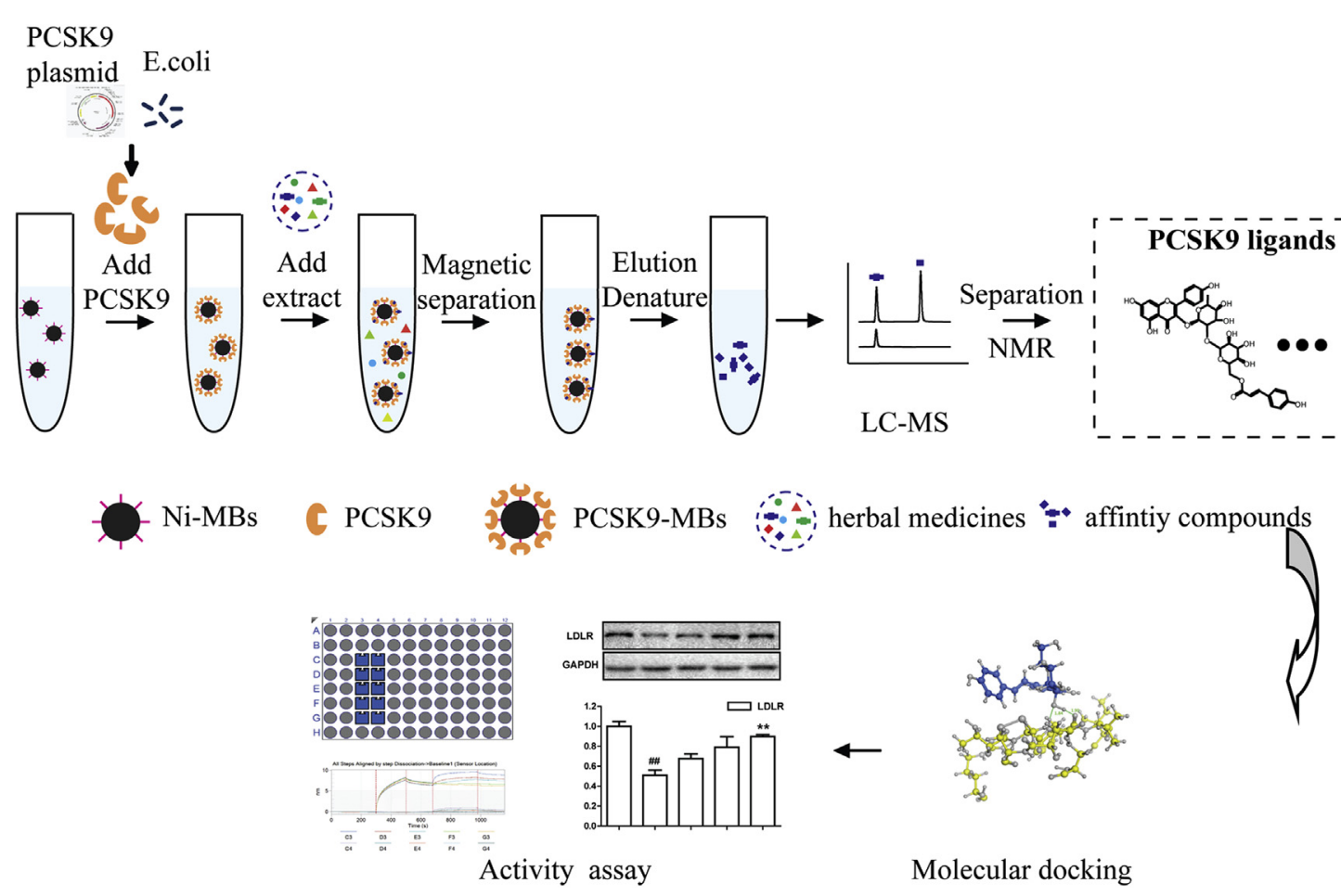

Fig. 1. The schematic diagram of the strategy for screening PCSK9 inhibitors from herbal medicines based on ligand fishing integrated interdisciplinary assay.

overnight. The cells were centrifuged at $4000 \mathrm{Xg}$ for $10 \mathrm{~min}$, and resuspended in $50 \mathrm{mM}$ sodium phosphate $(\mathrm{pH} 8.0) / 0.3 \mathrm{M} \mathrm{NaCl}$. The cell pellets were lysed by ultrasonication. After centrifuged, the lysate containing recombinant PCSK9 was obtained. Then, the lysate solution was flowed through a NiNTA resin column to purify the PCSK9 protein with a hexahistidine-tag (His-PCSK9), and the HisPCSK9 eluent was concentrated by ultrafiltration to $0.5 \mathrm{mg} / \mathrm{mL}$. All protein purification steps were conducted at $4{ }^{\circ} \mathrm{C}$ as quickly as possible. Whereafter, we chose Ni magnetic beads to link PCSK9 by the chelate action between $\mathrm{Ni}^{2+}$ and the His-tag. The Ni beads $(\sim 10 \mathrm{mg})$ was mixed with $1 \mathrm{~mL}$ PCSK9 solution $(50 \mu \mathrm{g} / \mathrm{mL})$, and then rotated together in a stirring mixer for $1 \mathrm{~h}$ at room temperature. Then, the PCSK9-MBs were separated by magnetism and rinsed by $100 \mu \mathrm{L}$ wash buffer (20 mM Sodium Phosphate, $500 \mathrm{mM} \mathrm{NaCl}, \mathrm{pH}$ 7.4) to exclude the non-specific adsorption. The PCSK9-MBs were stored in PBS at $4{ }^{\circ} \mathrm{C}$ for the next experiments. The stability of PCSK9-MBs within $72 \mathrm{~h}$ was evaluated.

\subsection{Optimization conditions for ligand screening}

The preparation of the PCSK9-MBs was as the above. To optimize the screening conditions, we selected the SBC-115076, a PCSK9 inhibitor, as the model drug [22]. The PCSK9-MBs $(100 \mu \mathrm{L})$ were incubated with SBC-115076 $(1 \mu \mathrm{L}, 100 \mathrm{mM})$ in PBS for a period to enable the ligand (SBC-115076) to bound to the PCSK9, and then the ligand-PCSK9MBs complexes were obtained by magnetic separation. The amount of SBC-115076 on the PCSK9MBs could be dissociated by adding $200 \mu \mathrm{L}$ methanol, followed by analysis by HPLC-MS. In this process, three parameters were optimized to obtain the appropriate screening conditions. Different ionic strength $(10,25,50,75$, and $100 \mathrm{mM})$ of PBS, incubation temperature $\left(4,16,25\right.$, and $\left.37^{\circ} \mathrm{C}\right)$ and incubation time $(0.5,1,2,4$, and $8 \mathrm{~h})$ were evaluated.

\subsection{Screening potential PCSK9 ligands from Ginkgo biloba leaves by LC/MS}

Ginkgo biloba leaves (GBL) were milled to powder. 1 g powdered GBL was ultrasonic extractedin $50 \mathrm{~mL} \mathrm{75 \%}$ $\mathrm{v} / \mathrm{v}$ methanol twice for $1 \mathrm{~h}$, and then filtered. The extracts of GBL were vacuum-evaporated to dryness and dissolved with $1 \mathrm{~mL}$ dimethyl sulfoxide (DMSO) to form the test solution. The test solution were store at $-20^{\circ} \mathrm{C}$.

After the PCSK9-MBs were prepared, the following process of ligand fishing consists of four steps: ligand 
adsorption, magnetic separation, ligand elution, and characterization. The procedure of ligand screening was conducted as the described above (Fig. 1). PCSK9MBs $(100 \mu \mathrm{L})$ was incubated with the extract of GBL $(1 \mu \mathrm{L})$ in the PBS $(200 \mu \mathrm{L}, 75 \mathrm{mM}, \mathrm{pH} 7.4)$ for $2 \mathrm{~h}$, and then conducted magnetic separation. The obtained ligand-PCSK9-MBs complexes were added $200 \mu \mathrm{L}$ methanol, shaked, filtered, and followed by analysis by HPLC-MS. Experiments were also set to use beads coating the denatured PCSK9 which were boiled in water for $10 \mathrm{~min}$. A blank control only incubated of PCSK9-MBs without the addition of GBL was conducted. The ligands would be captured by the ligand fishing and identified by HPLC-MS/MS.

HPLC-Q-TOF-MS/MS was performed on an Agilent 1260 series HPLC system coupled with an Agilent 6530 Q-TOF mass spectrometer equipped with an electrospray ionization (ESI) source. An Agilent ZORBAX SB-C18 column $(4.6 \mathrm{~mm} \times 250 \mathrm{~mm}, 5 \mu \mathrm{m})$ was used for sample separation. The flow rate was $0.8 \mathrm{~mL} / \mathrm{min}$, and the column temperature was maintained at $35{ }^{\circ} \mathrm{C}$. The mobile phase was water-formic acid (100:0.1, A) and acetonitrile (B) using a gradient elution program of 0-20 $\mathrm{min}, 10 \%-30 \% \mathrm{~B} ; 20-45 \mathrm{~min}, 30 \%-70 \% \mathrm{~B}$; $45-52 \mathrm{~min}, 70 \%-95 \% \mathrm{~B} ; 52-55 \mathrm{~min}, 95-95 \%$ B. The detection wavelengths were $203 \mathrm{~nm}, 254 \mathrm{~nm}$, $280 \mathrm{~nm}, 310 \mathrm{~nm}$, and $365 \mathrm{~nm}$. The sample volume injected was $20 \mu \mathrm{L}$.

The ESI-MS acquisition conditions were in negative modes set as follows: The mass range was scanned from $m / z$ 100-1500 with collision energy (CE) from $10 \mathrm{eV}$ to $50 \mathrm{eV} \mathrm{N}$ flow rate was set as $10.0 \mathrm{~L} / \mathrm{min}$ at $350{ }^{\circ} \mathrm{C}$; nebulizer pressure at $35 \mathrm{psig}$; capillary voltage at $3500 \mathrm{~V}$; OCT RFV at $750 \mathrm{~V}$; and fragmentor voltage at $120 \mathrm{~V}$. The TOF mass spectrometer was calibrated every day before sample analysis for guaranteeing mass accuracy. The data acquisition and analysis were controlled by Agilent Mass Hunter Workstation software version B.07.00.

\subsection{Molecular docking}

To rapidly predict the potential ability of ligands above, molecular docking of the ligand-target interaction was conducted with the Molecular Operating Environment (MOE.2009.10) software. The protein structure of PCSK9 was downloaded from the Protein Data Bank (PDB code: 3gcx). The compounds were drawn and protonated in MOE, and the three-dimensional structure of PCSK9 was removed the ligands, water molecules, and metal ions before docking. The compounds were docked into the binding sites of the PCSK9 respectively after the energy was minimized. The poses of molecules were obtained and scored using ASE scoring function.

\subsection{Cell culture}

HepG2 cells (ATCC) were grown in DMEM supplemented with $10 \%(\mathrm{v} / \mathrm{v})$ FBS, streptomycin $(50 \mathrm{~g} /$ $\mathrm{mL})$, L-glutamine $(2 \mathrm{mM})$ and penicillin $(50 \mathrm{U} / \mathrm{mL})$. HepG2 cells were incubated in six multi-well plates for $12 \mathrm{~h}$ at $37^{\circ} \mathrm{C}$. For different treatments, cells were divided into four groups. Before the addition to cells, ligands at different concentrations $(0,0.5,1.5$, $5.0 \mu \mathrm{M})$ were incubated with PCSK9 $(15 \mu \mathrm{g} / \mathrm{mL})$ for $30 \mathrm{~min}$ in the presence of $0.5 \%$ DMSO. Then, the cells above were incubated for $24 \mathrm{~h}$.

\subsection{Western blot analysis of cell LDL receptor}

Cells were lysed with RIPA lysis buffer and proteins were harvested. Total cell protein extracts were separated by $8 \%$ SDS-PAGE gel, and then transferred onto $0.22 \mu \mathrm{m}$ PVDF membranes (Millipore). The protein on PVDF membranes were blocked with $5 \%$ BSA-TBST at room temperature for $2 \mathrm{~h}$. Then, the PVDF membranes were incubated with anti-LDLR (1:4000; abcam) and anti-GAPDH (1:4000; Sigma) antibodies at $4{ }^{\circ} \mathrm{C}$ overnight, respectively. After washed three times with TBST, the membranes were then incubated with HRP-conjugated anti-rabbit IgG (1:4000; Cell Signaling Technology) for $2 \mathrm{~h}$ at room temperature. The immunoreactive bands were detected using ECL reagents by Bio-rad Gel Imaging System. The protein was quantified based on the intensity of bands by the Image Lab analysis software and normalized to the internal protein GAPDH. All experiments were performed in triplicate.

\subsection{Bio-layer interferometry binding assays}

Binding kinetics of the ligand-PCSK9 interaction were determined by the bio-layer interferometry (BLI) method. All experiments were conducted in PBS buffers ( $\mathrm{pH}$ 7.4) using Octet RED96 system (FortéBio) at $25{ }^{\circ} \mathrm{C}$. His-PCSK9 was immobilized onto Ni-NTA biosensors, and then the proteincoated biosensors were used to measure the process of association and dissociation with a time window of 300 and $180 \mathrm{~s}$, respectively. There were two control tests measured in parallel for double referencing corrections: protein-coated biosensors alone, and uncoated biosensors with compounds at corresponding concentrations. The binding constants were calculated by subtracted binding interference data with the FortéBio analysis software. 


\subsection{Statistical analysis}

All experiments were performed in triplicate. Statistical analysis was performed using GraphPad Prism 6.0 Software (San Diego, CA, USA). Data are presented as the mean \pm SEM. Group comparisons were assessed with the two-tailed Student's $t$-test or ANOVA with Bonferroni's post hoc test for comparison of multiple columns. A value of $P<0.05$ was considered as statistically significant.

\section{Results and discussion}

\subsection{Preparation and characterization of the PCSK9-MBs}

The main function of secreted PCSK9 is inducing the degradation of LDLR. The catalytic domain and the C-terminal domain of PCSK9 are two important domains for the PCSK9/LDLR interaction, leading to the degradation pathway of LDLR [23-26]. Considering the bioactive function of reconstituted His-PCSK9, the catalytic domain and C-terminal domain of human PCSK9 were selected to be expressed as targets for ligands fishing. As our previous study [22], the protein (His-PCSK9) was expressed and purified with a hexahistidine tag at the N-terminus (Fig. S1). The purified His-PCSK9 had the characterization for inducing LDLR degradation, which indicated that reconstituted HisPCSK9 maintained the bioactive function of the prototype PCSK9 (Fig. S2). Then, the His-PCSK9 were coupled to the magnetic beads as described above, and the preparation procedures of PCSK9MBs were repeatable. PCSK9-MBs could remain more than $72 \mathrm{~h}$ (Table S1).

\subsection{Optimization of ligand fishing conditions}

The ligand fishing method based on PCSK9MBs, was verified and optimized using one known compound, SBC-115076, which was the positive inhibitor of PCSK9. To guarantee the appropriate screening conditions of the active components in mixtures, three important parameters were optimized, including incubation time, incubation temperature and PBS ionic strength [27]. Incubation times between 0.5 and $8 \mathrm{~h}$ were tested using SBC-115076 (Fig. 2A). With excess SBC-115076, $2 \mathrm{~h}$ was the appropriate time for the ligands-protein interaction. Incubating for a long time may cause the denaturation of PCSK9 or disassembly of PCSK9 MBs, leading to lower binding degrees. Considering the properity of PCSK9 and the stability of PCSK9-MBs, the incubation $\mathrm{pH}$ was chosen as the normal physiological the $\mathrm{pH}$ 7.4. $25{ }^{\circ} \mathrm{C}$ was demonstrated as the most suitable temperature for the ligand binding (Fig. 2B). The ionic strength of the binding buffer may impact on the charge distribution on the protein surfaces. As shown in Fig. 2C, the proper ionic strength was $75 \mathrm{mM}$. With the increase of the ion strength, the concentration of ions was exceeded, which might reverse the effect of ions, resulting in lower binding degrees. From the above, the parameters were optimized as follows: incubation at $25{ }^{\circ} \mathrm{C}$ for $2 \mathrm{~h}$, and ionic concentration $75 \mathrm{mM}(\mathrm{pH}$ 7.4).

\subsection{PCSK9 ligand fishing of Ginkgo biloba leaves and HPLC-Q-TOF-MS/MS analysis}

According to the optimized conditions above, we screened the potential ligands of PCSK9 from GBL using HPLC-Q-TOF-MS/MS. The chromatograms of screening results were shown in Fig. 3. Compared with HPLC spectrums at different wavelengths, we chose $280 \mathrm{~nm}$ as the detection wavelength, which may reveal the difference between the peak areas with and without the PCSK9 incubation more distinct and undisturbed. The UV peak areas of two components were demonstrated to be significantly higher in the group incubated with PCSK9-MBs than the control group. Based on our strategy, these two components were regarded as the major potential ligands binding to the PCSK9, which were considered to apply for follow-up study. The affinity compounds were subsequently identified exactly by comparing with standards and published data. Considering that the intensities of negative ion signals exhibited higher than that of positive ion signals, we selected the negative ion mode for MS assay. The HPLC and MS data of two compounds (1 and 2) were listed in Table 1.

For Compound 1, it was identified as kaempferol3-O-rutinoside by compared with the standard compound (Fig. S3). The formula of compound 1 was calculated as $\mathrm{C}_{27} \mathrm{H}_{30} \mathrm{O}_{15}$ based on the molecular ion at $\mathrm{m} / \mathrm{z}$ 593.1505. The negative mode MS/MS product ions $m / z 447$ and 285 were assigned as [M$\mathrm{H}_{-R u t i n o s e}{ }^{-}$and $[\mathrm{M}-\mathrm{H}-\text { Rutinose-glu }]^{-}$. The other fragment ions were at $m / z 327$ ([M-H-Rutinose$\left.\mathrm{C}_{4} \mathrm{H}_{8} \mathrm{O}_{4}\right]^{-}$) and $m / z \quad 255$ ([M-H-Rutinoseglu- $\left.\left.\mathrm{CO}-\mathrm{H}_{2}\right]^{-}\right)$. The fragment ions $\mathrm{m} / \mathrm{z} 285$ could further produce ion $\mathrm{m} / \mathrm{z} 151$ by the retro-Diel$\mathrm{s}$-Alder (RDA) reaction. The formula of compound 2 was calculated as $\mathrm{C}_{36} \mathrm{H}_{36} \mathrm{O}_{17}$ based on the molecular ion at $\mathrm{m} / z$ 739.1889. In the MS/MS spectra, fragment ions at $m / z 593$ and 284 were produced by successive neutral losses of $146 \mathrm{Da}$ (Coumaroyl) and 
A

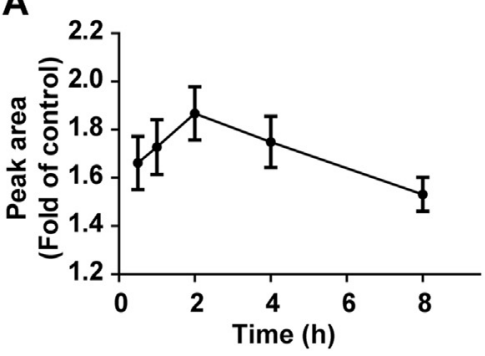

B

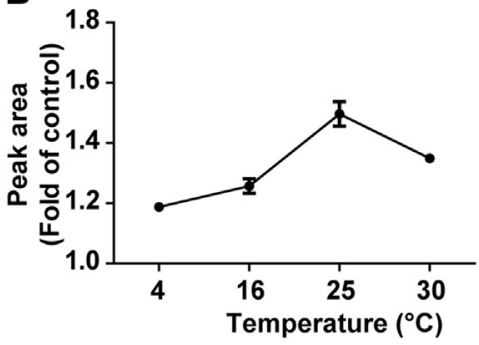

C

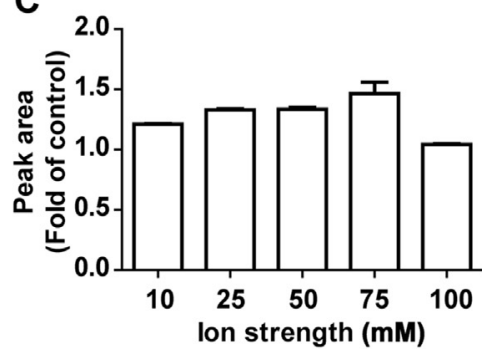

Fig. 2. The parameter optimization of the incubation time (A), temperature (B) and ionic strength of PBS (C) on the binding properties of ligands to PCSK9. SBC-115076 was the model compound.

$308 \mathrm{Da}(\mathrm{rha}+\mathrm{glu})$ (Fig. S4). The other fragment ions were at $\mathrm{m} / \mathrm{z} 255$ ([M-H-Coumaroyl-rhaglu- $\left.\mathrm{CO}-\mathrm{H}_{2}\right]^{-}$) and $\mathrm{m} / \mathrm{z} \quad 145$ ([Coumaroyl-H] $]^{-}$). Compound 2 was subsequently isolated and purified (Fig. S5), and determined with NMR (Fig. S6). By comparing the HPLC-MS/MS and NMR data with the reference [28], compound 2 was identified to be kaempferol 3-O-2"-(6"'-p-coumaroyl) glucosylrhamnoside (KCGR). The chemical structures of compound 1 and 2 were shown in Fig. 3B. They are both classified into flavonoid compounds. According to the previous study, the amount of compound

A
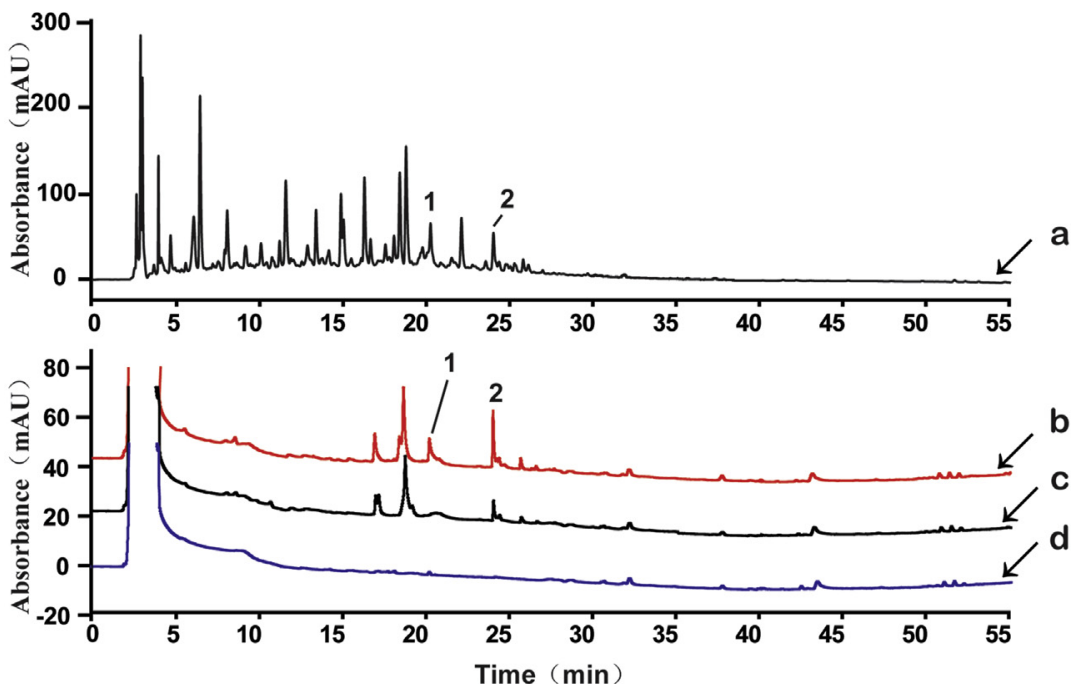

B<smiles>C[C@H]1O[C@H](OC[C@H]2O[C@H](Oc3c(-c4ccc(O)cc4)oc4cc(O)cc(O)c4c3=O)[C@@H](O)[C@H](O)[C@H]2O)C(O)[C@H](O)C1O</smiles><smiles>O=C(C=Cc1ccc(O)cc1)OCC1OC(OC2C(Oc3c(-c4ccc(O)cc4)oc4cc(O)cc(O)c4c3=O)OC(c3ccc(O)cc3)C(O)C2O)C(O)C(O)C1O</smiles>

2

Fig. 3. (A) The comparison HPLC chromatograms of the PCSK9 ligand fishing in the Ginkgo biloba leaves (GBL) at 280 nm. (a) represents the HPLC profile of the extract of GBL; (b) represents the HPLC profile when the GBL incubated with active PCSK9-MBs; (c) represents HPLC profile when the GBL incubated with inactive PCSK9-MBs; (d) represents the HPLC profile of the blank control which was only incubated of PCSK9-MBs without the addition of GBL. The screened ligands are labeled above the peaks with numbers. (B) Chemical structures of fished compounds 1 and 2, which were kaempferol-3-O-rutinoside and kaempferol 3-O-2"-(6"'-p-coumaroyl) glucosylrhamnoside (KCGR), respectively. 
Table 1. Retention times, UV absorption maxima and MS data of affinity compounds on PCSK9 from Ginkgo biloba leaves by HPLC-MS/MS.

\begin{tabular}{|c|c|c|c|c|c|c|c|c|}
\hline \multirow[t]{2}{*}{ Peak NO. } & \multirow[t]{2}{*}{ RT (min) } & \multirow[t]{2}{*}{ UVmax (nm) } & \multicolumn{3}{|l|}{$[\mathrm{M}-\mathrm{H}]-$} & \multirow[t]{2}{*}{ Fragment } & \multirow[t]{2}{*}{ Formula } & \multirow[t]{2}{*}{ Identification } \\
\hline & & & $m / z$ & $\mathrm{Cal} m / z$ & Diff (ppm) & & & \\
\hline 1 & 19.908 & 266,344 & 593.1505 & 593.1512 & 1.17 & $285.0397,151.0017$ & $\mathrm{C}_{27} \mathrm{H}_{30} \mathrm{O}_{15}$ & $\begin{array}{l}\text { Kaempferol 3-O-2"- } \\
\text { glucosylrhamnoside }\end{array}$ \\
\hline 2 & 23.627 & 266,316 & 739.1889 & 739.1880 & -1.25 & $\begin{array}{l}593.1510,413.0890, \\
284.0328,145.0290\end{array}$ & $\mathrm{C}_{36} \mathrm{H}_{36} \mathrm{O}_{17}$ & $\begin{array}{l}\text { Kaempferol 3-O-2"-(6"'-p- } \\
\text { coumaroyl) glucosylrhamnoside }\end{array}$ \\
\hline
\end{tabular}

[a] Compared with standard compounds.

1 and 2 present in the extract of Ginkgo biloba leaves were $13.08-15.06 \mathrm{mg} / \mathrm{g}$ and $20.12-25.21 \mathrm{mg} /$ g, respectively [29].

\subsection{Molecular docking}

Parallel assays by setting the control group could be applied to eliminate the false positive, but the repeated operation was indubitably inefficient. The molecular docking was promised to be alternated for excluding the non-specific affinity. Thus, we used the molecular docking assay to predict binding sites and potential activity of two possible ligands, which may contribute to seeking out the potential inhibitors of the PCSK9 rapidly. The results were evaluated according to the docking poses and the ligand-protein interactions [30]. The binding energies and affinity of ligands to PCSK9 were summarized in Table 2.

In consideration of binding energy and affinity, KCGR (compound 2) was preferred with significantly lower binding energy $(-14.4342 \mathrm{kcal} / \mathrm{mol})$ and higher affinity $(6.68 \mathrm{pKi})$ than the positive control SBC-115076 (-10.0054 kcal/mol, $5.83 \mathrm{pKi})$. The protein-ligand interaction between KCGR and the PCSK9 were presented in Fig. 4. Four hydrogen bonding were formed between KCGR (compound 2) and PCSK9 at the Asp 374, Ser 372 and Ser 221 (Fig. 4B). Besides, the other compound (compound 1) was also predicted to have certain interactions with PCSK9, such as Phe 379 and Thr 377 (Fig. S7), but its binding affinity was relatively inferior to that of KCGR and SBC-115076, which might possess slightly weak activity. The previous research has reported that the structures of kaempferol and $p$ coumaric acid might contribute to the PCSK9 inhibition [31], which was consistent with our research.

Table 2. The binding energies and affinity of PCSK9 ligands by molecular docking assay.

\begin{tabular}{lll}
\hline Name & Binding energies $(\mathrm{kcal} / \mathrm{mol})$ & Affinity $(\mathrm{pKi})$ \\
\hline SBC-115076 & -10.0054 & 5.83 \\
1 & -13.6614 & 5.07 \\
2 & -14.4342 & 6.68 \\
\hline
\end{tabular}

Moreover, our study further investigated and clarified the interaction between ligands and PCSK9.

From the above, with molecular docking, compounds 2 beared significant protein affinity. However, molecular docking assay is a virtual approach to predict the affinity between ligands and the target, so the activities of ligands were still needed to be confirmed by activity assays, which could also validate the established method.

\subsection{PCSK9 inhibitory activity of affinity fishing compounds}

The results of cell assay indicated that both compounds $1\left(\mathrm{IC}_{50}=0.4993 \mu \mathrm{M}\right)$ and $2\left(\mathrm{IC}_{50}=0.1837 \mu \mathrm{M}\right)$ could suppress the PCSK9-induced LDLR degradation in HepG2 cells in a concentration-dependent manner (Fig. 5). At the tested concentration of $5 \mu \mathrm{M}$, the expression of LDLR in the group incubated with compound 1 could restore to about $90 \%$ of control levels (Fig. 5B). Fortunately, compound 2 demonstrated higher activity than that of compound 1 . The LDLR levels in the group incubated with compound 2 were restored and reached higher than that in the control group at the concentration of $5 \mu \mathrm{M}$ (Fig. 5C). Compound 2 also showed the prominent inhibitory activity better than that of positive control SBC115076 (Fig. 5A), which reminded that it deserved further studied as the potential drug candidate. Kaempferol glycosides are very common natural products from plants, which were widely studied for their ability on improvement of the LDLR expression. The related study has reported that kaempferol could stimulate gene expression of LDLR through activation of Sp1 in cultured hepatocytes [32]. Besides this, a new mechanism might be proposed in our study about the kaempferol glycosides which could interact with PCSK9 for increasing the expression of LDLR.

Binding thermodynamic and kinetics have deemed as important design parameters for inhibitors. Therein, slow binding off-rates were considered to be related to improved inhibitor efficacies due to prolonged effects on the target in vivo [33]. So, we further detected the kinetic aspects of 
A

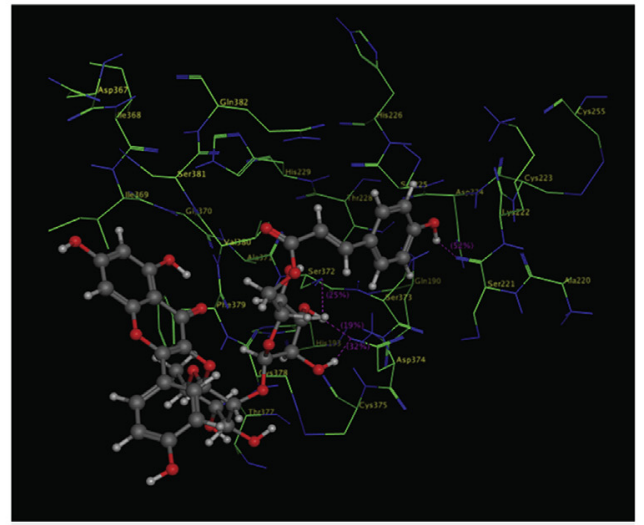

B

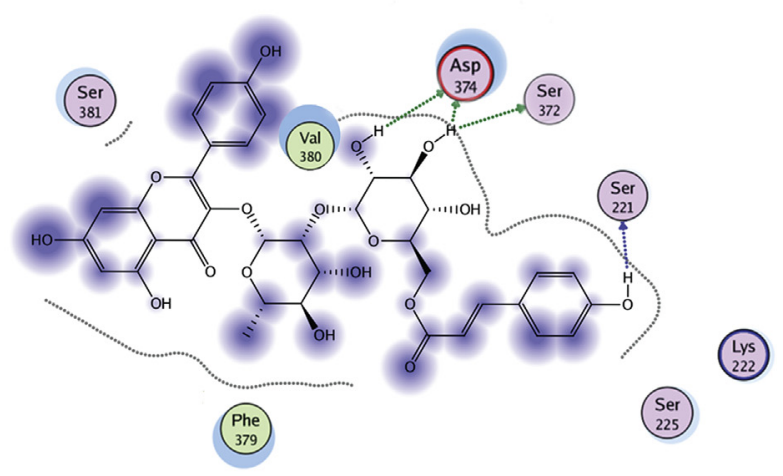

Fig. 4. The Spatial (A) and two-dimension (B) structures of the microenvironment interaction between KCGR and PCSK9 (PDB: $3 g c x)$ generated with MOE. The atom colors of the KCGR and the residues of PCSK9 were grey and green, respectively.

the compound 2 (KCGR) interacted with PCSK9 using bio-layer interferometry (BLI) analysis. BLI experiments were conducted at $\mathrm{pH} 7.4$ and the $\mathrm{K}_{\mathrm{D}}$ values for KCGR was obtained. Interestingly, we observed slow dissociation rates of KCGR when binding to PCSK9 (Fig. 5D). Commonly, slower kinetics has been considered as a characteristic of some type-II allosteric inhibitors possibly due to kinetic constrains of the necessary structural rearrangements [34,35], resulting in $K_{D}$ values in the low nanomolar range $\left(K_{D}=15 \mathrm{nM}\right)$. The low $K_{D}$ value reminder that compound 2 (KCGR) could be considered as a candidate PCSK9 inhibitor for the pharmaceutical development.
A
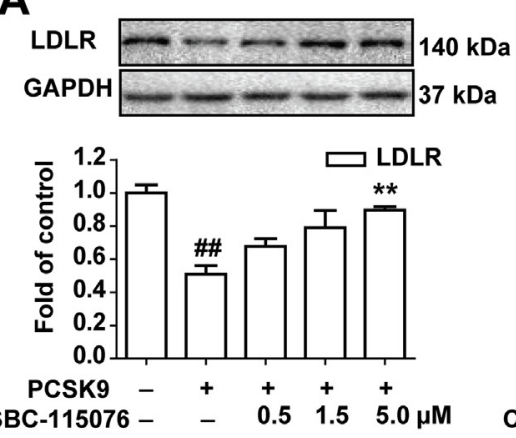

B
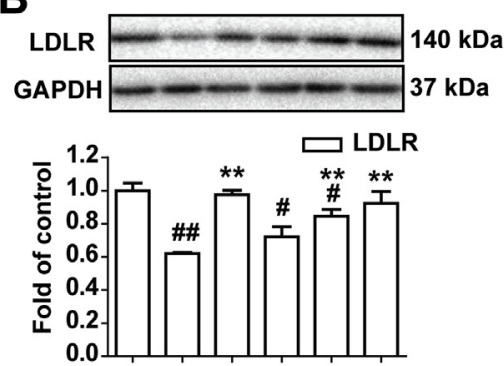

PCSK9 - + -+++ Compound $1-\quad-\quad 1.50 .51 .55 .0 \mu \mathrm{M}$
C
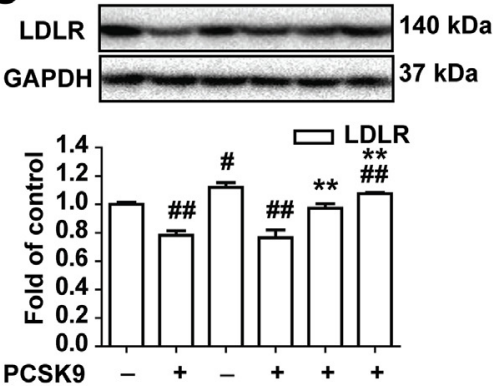

D

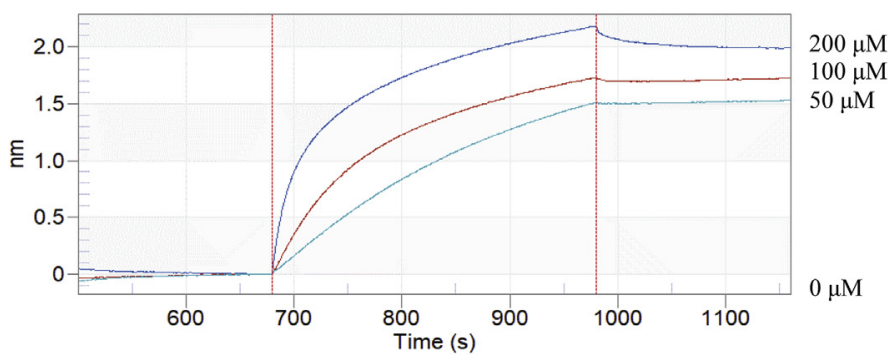

Fig. 5. The inhibitory activities of the fished compounds on PCSK9. The potency of SBC-115076 (A), compound 1 (B) and compound 2 (C) for restoring LDLR levels on the PCSK9-treated HepG2 cells. The expression levels of LDLR on HepG2 cells were semiquantitated by Western blot assay. The values are the mean \pm SEM deviation of the three independent experiments. \#p $<0.05 ; \#$ \# $<0.01 ;$ compared with the control group, * $p<0.05$; $* * p<0.01$, compared with the PCSK9 group. (D). The BLI sensogram showing the process of association and dissociation at different concentrations $(0,50,100$, and $200 \mu M)$ for the interaction of compound 2 (KCGR) with PCSK9. 


\section{Conclusions}

In conclusion, an effective strategy integrating ligand fishing, molecular docking, binding assay and inhibitory activity assay was developed for screening PCSK9 inhibitors from GBL, which was demonstrated to be valid and practicable. On the basis of our strategy, two compounds were fished out from GBL, including kaempferol 3-O-2"-glucosylrhamnoside (compound 1) and kaempferol 3-O2"-(6"' -p-coumaroyl) glucosylrhamnoside (KCGR, compound 2). KCGR was determined to exhibit significant inhibitory activity against PCSK9. Our study provides the basis for further exploration of active compounds from GBL in the intervention and prevention of the PCSK9-related diseases for future clinical use. Moreover, with the properties of targeted screening and accurate analysis, this strategy is supposed to be further served for an extended range to rapidly screen active ingredients from mixtures, which will expedite the efficiency of drug discovery.

\section{Conflict of interest}

The authors declare that there are no conflicts of interest.

\section{Acknowledgments}

This study was supported by the Priority Academic Program Development of Jiangsu Higher Education Institutions (PAPD).

\section{Appendix A. Supplementary data}

Supplementary data to this article can be found online at https://doi.org/10.38212/2224-6614.1061.

\section{References}

[1] He XX, Zhang R, Zuo PY, Liu YW, Zha XN, Shan SS, et al. The efficacy advantage of evolocumab (AMG 145) dosed at $140 \mathrm{mg}$ every 2 weeks versus $420 \mathrm{mg}$ every 4 weeks in patients with hypercholesterolemia: evidence from a meta-analysis. Eur J Intern Med 2017;38:52-60.

[2] Puri R, Nissen SE, Somaratne R, Cho L, Kastelein JJ, Ballantyne CM, et al. Impact of PCSK9 inhibition on coronary atheroma progression: rationale and design of global assessment of plaque regression with a PCSK9 antibody as measured by intravascular ultrasound (GLAGOV). Am Heart J 2016;176:83-92.

[3] Bos S, Duvekot MH, Ten Kate GR, Verhoeven AJ, Mulder MT, Schinkel AF, et al. Carotid artery plaques and intima medial thickness in familial hypercholesteraemic patients on long-term statin therapy: a case control study. Atherosclerosis 2017;256:62-6.

[4] Versmissen J, Oosterveer DM, Yazdanpanah M, Defesche JC, Basart DC, Liem AH, et al. Efficacy of statins in familial hypercholesterolaemia: a long term cohort study. BMJ 2008; 337:a2423.

[5] Steinberg D, Witztum JL. Inhibition of PCSK9: a powerful weapon for achieving ideal LDL cholesterol levels. Proc Natl Acad Sci U S A 2009;106:9546-7.

[6] Li J, Tumanut C, Gavigan JA, Huang WJ, Hampton EN, Tumanut R, et al. Secreted PCSK9 promotes LDL receptor degradation independently of proteolytic activity. Biochem J 2007:406:203-7.

[7] Lagace TA, Curtis DE, Garuti R, McNutt MC, Park SW, Prather HB, et al. Secreted PCSK9 decreases the number of LDL receptors in hepatocytes and in livers of parabiotic mice. J Clin Invest 2006;116:2995-3005.

[8] Abifadel M, Elbitar S, El Khoury P, Ghaleb Y, Chemaly M, Moussalli ML, et al. Living the PCSK9 adventure: from the identification of a new gene in familial hypercholesterolemia towards a potential new class of anticholesterol drugs. Curr Atherosclerosis Rep 2014;16:439.

[9] Yadav K, Sharma M, Ferdinand KC. Proprotein convertase subtilisin/kexin type 9 (PCSK9) inhibitors: present perspectives and future horizons. Nutr Metabol Cardiovasc Dis 2016; 26:853-62.

[10] Ramanathan A, Gusarova V, Stahl N, Gurnett-Bander A, Kyratsous CA. Alirocumab, a therapeutic human antibody to PCSK9, does not affect CD81 levels or hepatitis C virus entry and replication into hepatocytes. PloS One 2016;11:e0154498.

[11] Zhou W, Chai H, Lin PH, Lumsden AB, Yao Q, Chen C. Clinical use and molecular mechanisms of action of extract of Ginkgo biloba leaves in cardiovascular diseases. Cardiovasc Drug Rev 2004;22:309-19.

[12] Singh B, Kaur P, Gopichand, Singh RD, Ahuja PS. Biology and chemistry of Ginkgo biloba. Fitoterapia 2008;79:401-18.

[13] Zhang Q, Wang GJ, JY A, Wu D, Zhu LL, Ma B, et al. Application of GC/MS-based metabonomic profiling in studying the lipid-regulating effects of Ginkgo biloba extract on diet-induced hyperlipidemia in rats. Acta Pharmacol Sin 2009;30:1674-87.

[14] Barner JC, Worchel J, Min Y. Frequency of new-onset diabetes mellitus and use of antipsychotic drugs among central Texas veterans. Pharmacotherapy 2004;24:1529-38.

[15] Yao P, Song F, Li K, Zhou S, Liu S, Sun X, et al. Ginkgo biloba extract prevents ethanol induced dyslipidemia. Am J Chin Med 2007;35:643-52.

[16] Wang XY, Ding X, Yuan YF, Zheng LY, Cao Y, Zhu ZY, et al. Comprehensive two-dimensional APTES-decorated MCF7cell membrane chromatographic system for characterizing potential anti-breast-cancer components from Yuanhu-Baizhi herbal medicine pair. J Food Drug Anal 2018;26:823-33.

[17] van Breemen RB, Huang CR, Nikolic D, Woodbury CP, Zhao YZ, Venton DL. Pulsed ultrafiltration mass spectrometry: a new method for screening combinatorial libraries. Anal Chem 1997;69:2159-64.

[18] Li L, Kong J, Yao CH, Liu XF, Liu JH. Rapid identification of urokinase plasminogen activator inhibitors from Traditional Chinese Medicines based on ultrafiltration, LC-MS and in silico docking. J Pharmaceut Biomed Anal 2019;164:241-8.

[19] Wang YJ, Lee SC, Hsu CH, Kuo YH, Yang CC, Lin FJ. Antcins, triterpenoids from Antrodia cinnamomea, as new agonists for peroxisome proliferator-activated receptor alpha. J Food Drug Anal 2019;27:295-304.

[20] de Almeida FG, Vanzolini KL, Cass QB. Angiotensin converting enzyme immobilized on magnetic beads as a tool for ligand fishing. J Pharmaceut Biomed Anal 2017;132:159-64.

[21] Wang Z, Li X, Chen M, Liu F, Han C, Kong L, et al. A strategy for screening of alpha-glucosidase inhibitors from Morus alba root bark based on the ligand fishing combined with high-performance liquid chromatography mass spectrometer and molecular docking. Talanta 2018;180:337-45.

[22] Li L, Shen C, Huang YX, Li YN, Liu XF, Liu XM, et al. A new strategy for rapidly screening natural inhibitors targeting the PCSK9/LDLR interaction in vitro. Molecules 2018;23. 
[23] Benjannet S, Rhainds D, Essalmani R, Mayne J, Wickham L, Jin $W$, et al. NARC-1/PCSK9 and its natural mutants: zymogen cleavage and effects on the low density lipoprotein (LDL) receptor and LDL cholesterol. J Biol Chem 2004;279: $48865-75$.

[24] Maxwell KN, Breslow JL. Adenoviral-mediated expression of Pcsk9 in mice results in a low-density lipoprotein receptor knockout phenotype. Proc Natl Acad Sci U S A 2004;101: 7100-5.

[25] Park SW, Moon YA, Horton JD. Post-transcriptional regulation of low density lipoprotein receptor protein by proprotein convertase subtilisin/kexin type 9a in mouse liver. J Biol Chem 2004;279:50630-8.

[26] Yamamoto T, Lu C, Ryan RO. A two-step binding model of PCSK9 interaction with the low density lipoprotein receptor. J Biol Chem 2011;286:5464-70.

[27] Papaneophytou CP, Grigoroudis AI, McInnes C, Kontopidis G. Quantification of the effects of ionic strength, viscosity, and hydrophobicity on protein-ligand binding affinity. ACS Med Chem Lett 2014;5:931-6.

[28] Song HP, Wang H, Liang JX, Qian C, Wu SQ, Xu WJ, et al. Integration of multiple analytical and computational tools for the discovery of high-potency enzyme inhibitors from herbal medicines. ChemMedChem 2016;11:2588-97.

[29] Wang JH, Chen J, Wang MM, Fu XT, Chen YG, Guo HZ. Determination of 7 flavonol glycosides in Ginkgo biloba reference extract. Zhongguo Zhongyao Zazhi 2015;40: 4018-21.
[30] Song HP, Chen J, Hong JY, Hao H, Qi LW, Lu J, et al. A strategy for screening of high-quality enzyme inhibitors from herbal medicines based on ultrafiltration LC-MS and in silico molecular docking. Chem Commun (Camb) 2015;51: 1494-7.

[31] Choi HK, Hwang JT, Nam TG, Kim SH, Min DK, Park SW, et al. Welsh onion extract inhibits PCSK9 expression contributing to the maintenance of the LDLR level under lipid depletion conditions of HepG2 cells. Food Funct 2017;8: 4582-91.

[32] Ochiai A, Miyata S, Iwase M, Shimizu M, Inoue J, Sato R. Kaempferol stimulates gene expression of low-density lipoprotein receptor through activation of $\mathrm{Sp} 1$ in cultured hepatocytes. Sci Rep 2016;6:24940.

[33] Wood ER, Truesdale AT, McDonald OB, Yuan D, Hassell A, Dickerson $\mathrm{SH}$, et al. A unique structure for epidermal growth factor receptor bound to GW572016 (Lapatinib): relationships among protein conformation, inhibitor off-rate, and receptor activity in tumor cells. Canc Res 2004;64:6652-9.

[34] Gruenbaum LM, Schwartz R, Woska Jr JR, DeLeon RP, Peet GW, Warren TC, et al. Inhibition of pro-inflammatory cytokine production by the dual p38/JNK2 inhibitor BIRB796 correlates with the inhibition of p38 signaling. Biochem Pharmacol 2009;77:422-32.

[35] Pargellis C, Tong L, Churchill L, Cirillo PF, Gilmore T, Graham AG, et al. Inhibition of p38 MAP kinase by utilizing a novel allosteric binding site. Nat Struct Biol 2002;9:268-72. 